\title{
No effect of vitamin $D$ supplementation on serum fibrinogen concentrations in adults aged $\geq 64$ years
}

\author{
M. S. Barnes ${ }^{1}$, L. K. Forsythe ${ }^{1}$, G. Horigan $^{1}$, M. P. Bonham ${ }^{1}$, E. M. Duffy ${ }^{1}$, T. R. Hill ${ }^{2}$, A. J. Lucey ${ }^{2}$, \\ K. D. Cashman ${ }^{2}$, M. Kiely ${ }^{2}$, J. J. Strain ${ }^{1}$ and J. M. W. Wallace \\ ${ }^{1}$ Northern Ireland Centre for Food \& Health (NICHE), University of Ulster, Coleraine BT52 ISA and ${ }^{2}$ Department of \\ Food and Nutritional Sciences, University College Cork, Republic of Ireland
}

High serum concentration of the acute phase protein fibrinogen is associated with tissue inflammation and is an independent risk factor for cardiovascular disease (CVD). Low vitamin D status is associated with an increased risk of CVD and the active form of the vitamin, 1,25dihydroxyvitamin $\mathrm{D}_{3}$, is a potent immunomodulator. Furthermore, vitamin $\mathrm{D}$ supplementation has been shown to reduce serum concentrations of inflammatory markers such as C-reactive protein in vitamin D deficient individuals. The aim of this study was to assess the effect of vitamin D supplementation on serum fibrinogen concentrations in a group of apparently healthy adults aged $\geq 64$ years recruited in Cork and Coleraine.

A total of 202 individuals (males, $n=81$; females, $n=121$ ) were randomly assigned to receive either 5,10 or $15 \mu \mathrm{g}$ vitamin $\mathrm{D}_{3} / \mathrm{d}$ or placebo for 22 weeks. Serum vitamin D status (25-hydroyvitamin D $(25(\mathrm{OH}) \mathrm{D}))$ and fibrinogen concentrations were measured at baseline and post intervention using commercially available ELISA kits.

Vitamin D status did not significantly correlate with serum fibrinogen concentrations at baseline or post intervention. One-way analysis of covariance (adjusted for age, sex, centre, body mass index and baseline concentrations) revealed that while vitamin D supplementation significantly increased vitamin D status, it did not alter fibrinogen concentrations.

\begin{tabular}{|c|c|c|c|c|c|c|c|c|c|}
\hline & \multicolumn{9}{|c|}{ Treatment group $\left(\mu \mathrm{g}\right.$ vitamin $\left.\mathrm{D}_{3} / \mathrm{d}^{*}\right)$} \\
\hline & \multicolumn{2}{|c|}{ Placebo $(n=54)$} & \multicolumn{2}{|c|}{$5 \mu \mathrm{g} / \mathrm{d}(n=48)$} & \multicolumn{2}{|c|}{$10 \mu \mathrm{g} / \mathrm{d}(n=52)$} & \multicolumn{2}{|c|}{$15 \mu \mathrm{g} / \mathrm{d}(n=48)$} & \multirow[t]{2}{*}{$P^{\dagger}$} \\
\hline \multicolumn{9}{|c|}{$25(\mathrm{OH}) \mathrm{D}(\mathrm{nmol} / \mathrm{l})$} & \\
\hline Pre & 59.07 & $(43.37,78.64)$ & 51.84 & $(40.28,71.34)$ & 55.53 & $(43.00,72.26)$ & 55.09 & $(39.39,70.82)$ & \\
\hline Post & 42.59 & $(27.82,55.86)^{\mathrm{a}}$ & 53.19 & $(45.57,68.73)^{\mathrm{b}}$ & 70.32 & $(57.98,81.81)^{\mathrm{cd}}$ & 73.86 & $(61.87,90.20)^{\mathrm{cd}}$ & $<0.0001$ \\
\hline \multicolumn{10}{|c|}{ Fibrinogen $(\mathrm{g} / \mathrm{l})$} \\
\hline Pre & 1.86 & $(1.25,2.91)$ & 2.12 & $(1.44,3.54)$ & 1.78 & $(1.25,2.85)$ & 2.22 & $(1.31,3.08)$ & \\
\hline Post & 2.19 & $(1.60,3.07)$ & 2.10 & $(1.47,3.79)$ & 2.02 & $(1.40,2.81)$ & 2.18 & $(1.74,3.20)$ & 0.889 \\
\hline
\end{tabular}

25(OH)D, 25-hydroxyvitamin D.

* Values are median (IQR).

$\dagger$ Effect of treatment assessed on log transformed data by ANCOVA. Different superscript letters denote significant differences between treatment groups (ANOVA).

In conclusion, vitamin D supplementation had a significant dose-response effect on vitamin D status, but did not affect serum concentrations of the inflammatory marker fibrinogen in healthy older adults. These findings concur with previous research in vitamin $\mathrm{D}$ deficient adults. However, it has been suggested that $25(\mathrm{OH}) \mathrm{D}$ concentrations $>100 \mathrm{nmol} / \mathrm{l}$ may be required for modulation of immune responses; concentrations higher than those observed in the current study, even after vitamin D supplementation.

This work was supported by the UK Food Standards Agency. 